\title{
Otra manera (diferente) de explicar la Teoría de Juegos
}

\section{Another (different) way to explain Theory of Games}

José Antonio Molina-Toucedo

ORCID: https://orcid.org/0000-0003-3589-836X

Universidad de Sevilla

Departamento de Análisis Económico

y Economía Política

jamolina@us.es

Fecha recepción: 21/11/2010

Fecha aceptación: 25/11/2019

DOI: http://dx.doi.org/10.12795/9788447221912.105

Pp.: 2356-2373 
Resumen

Esta comunicación tiene como objetivo presentar y analizar un plan de mejora e innovación docente en la asignatura Microeconomía II (2o Grado de Economía de la Facultad de CC.EE y EE. de la Universidad de Sevilla). El objetivo es profundizar en la metodología docente cognitivista y constructivista, aplicando Aprendizaje Basado en Problemas (ABP) a través de un taller conceptual sobre cuatro microcasos de teoría de juegos. Se elabora una encuesta inicial para conocer los modelos mentales de los estudiantes y calibrar el gradiente de dificultad del objeto de estudio. Tras aplicar una metodología de taller conceptual, con dinámica de trabajo por equipos, se procede a la realización de la encuesta final. Los resultados obtenidos son alentadores.

Palabras clave: Microeconomía, grado en economía, docencia universitaria, experimentación docente universitaria, teoría de juegos.

\section{Abstract}

This communication aims to implement a plan for improvement and teaching innovation in the subject Microeconomics II (2nd Degree of Economics of the Faculty of CC.EE and EE. of the University of Seville). The objective is to deepen the cognitive and constructive teaching methodology, applying Problem Based Learning (PBL) through a conceptual workshop on four micro cases of theory of games. An initial survey is prepared to know the students' mental models and calibrate the difficulty gradient of the object of study. After applying a conceptual workshop methodology with teamwork dynamics, the final survey is carried out. The results obtained are encouraging.

Keywords: Microeconomics, degree in economics, university teaching, university teaching experimentation, theory of games.

Jornadas de Formación e Innovación Docente del Profesorado | № 2 (2019) Esta obra se distribuye con la licencia Creative Commons 


\section{Descripción del contexto de la asignatura}

Este contenido es parte del Proyecto Docente de la asignatura Microeconomía II de 2o. Es una asignatura básica del primer cuatrimestre del Grado de Economía de la Universidad de Sevilla, que consta de 6 créditos CTS y un un total de 3 grupos, 2 de mañana y 1 de tarde. El Grupo M1 tiene en el Curso 2019-20 un total de 57 alumnos matriculados. El lugar es el Aula 25 de la Facultad de CC.EE. y $\mathrm{EE}$, que consta de 86 plazas, dispuestas en forma de grada con pendiente. El aula cuenta con cañón, ordenador y servicio de megafonía. Se ha escogido como objeto de estudio del Ciclo de Mejora Docente (Porlan, 2017) (CMD a partir de ahora) la toma de decisiones económicas (Introducción a la Teoría de Juegos). La finalidad de la enseñanza es hacer reflexionar a los alumnos sobre las maneras de tomar decisiones en asuntos económicos. Es decir, los distintos puntos de vista desde los que podemos enfocar nuestros análisis, deducciones y finalmente nuestras decisiones.

\section{Diseño previo del CMD y modelo metodológico posible}

\section{Mapa de contenidos}

La Toma de Decisiones en economía ha ido incorporando distintas teorías que han enriquecido el contenido epistemológico sobre esta materia. La Escuela de Pensamiento Clásica de finales del s. XVIII aportó la visión egocéntrica de que el "propio interés llevaba al interés común". A mediados del S. XX Von Neumann y Morgenstern publicaron su teoría de juegos y comportamiento social (Von Neumann, 2007). En donde introdujeron el planteamiento estratégico en los juegos no cooperativos o competitivos, en el que la acción de cada jugador se ve influida e influye en la de los demás jugadores. Por último, pocos años 
después John Nash, aporta la idea de la dinámica rectora, por la que cada jugador de un juego no cooperativo debe de elegir lo mejor en función de la elección de los demás jugadores (Nash, 1950).

En la figura 1 se presenta el mapa de contenidos elaborado para abordar el objeto de estudio: la toma de decisiones en economía. Están representados las tres grandes aportaciones conceptuales: enfoque clásico, teoría de juegos y dinámica rectora. El primero es el más básico y no presenta distinción entre juegos cooperativos y no cooperativos. Los otros dos sí lo hacen e interactúan entre sí en la categoría de los juegos no cooperativos. Los contenidos procedimentales aportan la conexión con la realidad en cualquiera de sus tres versiones, de menor a mayor complejidad: anécdotas económicas, microcasos y casos.

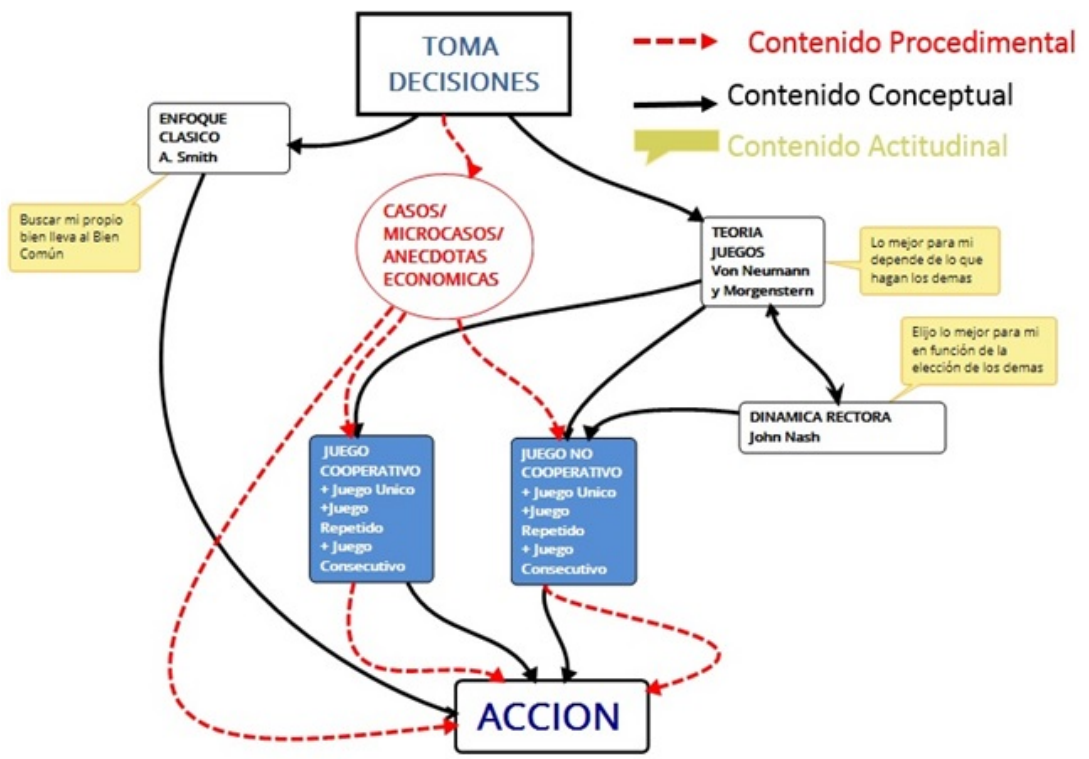

Figura 1. Mapa de contenidos conceptuales, procedimentales y actitudinales

Jornadas de Formación e Innovación Docente del Profesorado | № 2 (2019) Esta obra se distribuye con la licencia Creative Commons Reconocimiento-NoComercial-SinObraDerivada 4.0 Internacional (CC BY-NC-ND 4.0.) 


\section{Modelo metodológico posible}

Se ha utilizado un modelo metodológico (Porlan, 2017) "inverso" al habitualmente usado. Es decir, comenzamos con varios Problemas Intrigantes iniciales sobre la tomas de decisiones con contenido económico, propuestas de forma individual en la encuesta inicial. A continuación se han realizado Actividades de Contraste de otros microcasos planteados a resolución en dinámica de grupos. Seguidamente se ha procedido a la explicación de la Teoría, analizando y resolviendo entre todos los microcasos. Finalmente se han vuelto a plantear los Problemas Intrigantes iniciales, analizándose posteriormente la correcta resolución y extrayendo las Conclusiones sobre el comportamiento más adecuado.

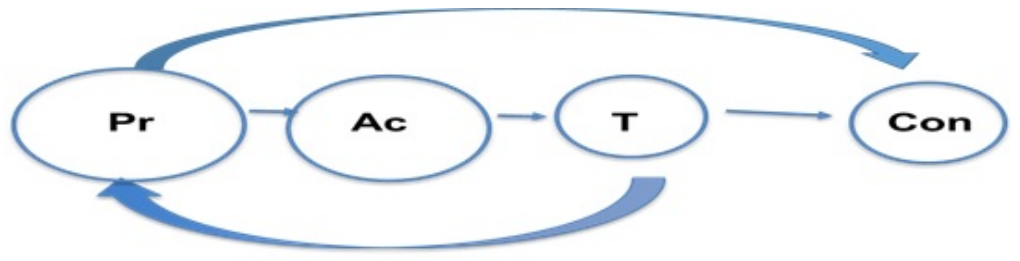

Figura 2. Modelo metodológico posible aplicado

\section{Secuencia de actividades}

Teniendo en cuenta el mapa de contenidos a enseñar y siendo coherente con el modelo metodológico que acabamos de presentar, diseñamos la secuencia de actividades que recoge la Tabla 1. 
Tabla 1. Extracto secuencia actividades

\begin{tabular}{|c|c|c|c|}
\hline SESIÓN & TIPO ACTIVIDAD & DESCRIPCIÓN Y RECURSOS & TIEMPO \\
\hline Primera & $\begin{array}{l}\text { Presentación. } \\
\text { Encuesta } \\
\text { Individual (Kahoot) } \\
\text { con } 6 \text { Problemas } \\
\text { Intrigantes. } \\
\text { Dinámica Grupo } \\
\text { con } 2 \text { microcasos }\end{array}$ & $\begin{array}{l}\text { En esta sesión se realizará la } \\
\text { Encuesta Inicial Individual. } \\
\text { Compuesta por } 10 \text { preguntas } \\
\text { ( } 5 \text { de control y } 5 \text { problemas } \\
\text { intrigantes). A continuación } \\
\text { una sesión de Trabajo en } \\
\text { Grupo con } 2 \text { microcasos. }\end{array}$ & $120 \mathrm{~m}$. \\
\hline $\begin{array}{l}\text { Segunda } \\
\text { y Tercera }\end{array}$ & $\begin{array}{l}\text { Resolución de } \\
\text { microcasos en } \\
\text { dinámica de } \\
\text { grupo. Explicación } \\
\text { teórica. Debate de } \\
\text { resultados }\end{array}$ & $\begin{array}{l}\text { En cada una de estas } 2 \\
\text { sesiones se presentaron } 4 \\
\text { microcasos de teoría de juegos } \\
\text { diferentes. Los microcasos } \\
\text { se plantearán uno a uno, } \\
\text { estipulando un tiempo de } \\
\text { discusión en los grupos y } \\
\text { posteriormente se debatirán } \\
\text { en común y se apuntarán } \\
\text { los resultados en la pizarra. } \\
\text { Seguidamente se explicarán } \\
\text { los contenidos teóricos y se } \\
\text { abrirá un debate sobre los } \\
\text { resultados aportados por los } \\
\text { grupos. }\end{array}$ & $240 \mathrm{~m}$. \\
\hline Cuarta & $\begin{array}{l}\text { Realización de la } \\
\text { Encuesta Inicial de } \\
\text { nuevo (Kahoot). } \\
\text { Debate posterior } \\
\text { abierto a } \\
\text { las posibles } \\
\text { soluciones }\end{array}$ & $\begin{array}{l}\text { Se volverá a realizar la } \\
\text { encuesta inicial con Kahoot. A } \\
\text { continuación se procederá a } \\
\text { debatir sobre la resolución de } \\
\text { los } 5 \text { problemas intrigantes. } \\
\text { El docente actúa como } \\
\text { moderador e inductor a } \\
\text { la reflexión con preguntas } \\
\text { incisivas sobre los aspectos } \\
\text { claves para superar los errores } \\
\text { conceptuales. }\end{array}$ & $120 \mathrm{~m}$. \\
\hline
\end{tabular}

\section{Cuestionarios inicial-final}

Para comprobar los modelos mentales de los alumnos respecto a la toma de decisiones, se ha elaborado un Cuestionario Inicial en la aplicación de gamificación 
Kahoot. Dicho cuestionario consta de un total de 9 preguntas. Las 5 primeras son preguntas informativas (de control) sobre el universo de los encuestados. De ellas, es reseñable la pregunta 3, que delimita que el número de repetidores de la asignatura en la muestra de alumnos es solamente uno (un porcentaje pequeño que evita una posible distorsión en los resultados del experimento). Las 4 restantes son preguntas desafiantes, cerradas y de opción múltiple, sobre decisiones de incertidumbre en toma de decisiones. El primer reto intrigante trata la equidad de un juego. La pregunta 7 plantea un Equilibrio de Nash. En el siguiente problema desafiante además de ponderar las consecuencias de las estrategias del competidor, la decisión correcta exigía valorar los intereses de los consumidores. Finalmente en la pregunta 9 se cuestiona sobre el resultado en un juego repetido. Es decir, hay que proyectar el comportamiento de los jugadores en un juego de infinitas jugadas. Posteriormente, como finalización del CMD se procedió a realizar la misma encuesta a modo de Cuestionario Final. La comparativa de éste cuestionario con el Inicial ha permitido analizar la evolución en el conocimiento de los alumnos, aplicando la "hipótesis de progresión" (HP) (García, 1998).

El Cuestionario Inicial fue respondido por un total de 38 alumnos y el Cuestionario Final por 36 alumnos.

\section{a) Preguntas datos alumnos:}

Pregunta 1. Soy: Mujer/Hombre

Pregunta 2. Mi franja de edad es: 1-19/20-21/22-25/>25.

Pregunta 3. ¿Es la primera vez que estudias la asignatura Microeconomía II?: Si/ No

Pregunta 4. Nací en: Sevilla y provincia/ Resto Andalucía/ Resto España/Resto Europa.

Pregunta 5. ¿Cómo sueles tomar las decisiones económicas?: Por intuición/Analizando mis Costes y Beneficios individuales/Analizando lo que hacen los demás/Analizando lo que hacen los demás y cómo me afecta a mí. 


\section{b) Preguntas intrigantes:}

Pregunta 6. Subasta de un billete de $1 \$$, a una sola puja. El mejor postor se lleva el billete. El segundo mejor postor también paga su oferta.¿Cuanto apuestas?: 0,5\$/1\$/0,25\$/ Nada.

Pregunta 7. Se plantea una matriz de beneficios para 2 empresas A y B que presentan 2 estrategias (hacer publicidad, no hacer publicidad): 10,5/ 15,0/ 6,8/ 20,2.

Pregunta 8. En esta plaza rectangular se van a emplazar el Bar Pepe y el Bar Paco, ¿dónde se situarán para maximizar beneficios?: uno frente al otro/ los dos pegados/ uno en medio y el otro en la esquina/ Ninguno es correcto.

Pregunta 9. Dada esta matriz de beneficios de la empresas A y B, que presentan dos estrategias (precio bajo, precio alto), ¿cuál va a ser resultado de este juego repetido?: 10,10/ 100, -50/ -50,100/ 50,50.

\section{Aplicación del CMD}

\section{Desarrollo}

La actividad se desarrolló en un total de 4 sesiones de 2 horas cada una de ellas, los días 21, 24, 28 y 31 de Octubre de 2019. Según el siguiente guion:

Sesión 1ạ (120 minutos)

Se les presentó el Modelo Metodológico, en el que comenzamos con la Encuesta Individual compuesta por un total de 9 preguntas que se ha presentado en el apartado 2.4. Los 38 alumnos que participaron son informados de que están realizando juegos simultáneos a una sola jugada en 4 de las ocasiones y un juego repetido de infinitas jugadas (pregunta 9). Para la ejecución de la encuesta se ha utilizado la herramienta de gamificación Kahoot, en 
su modalidad de encuesta (survey) y en el modo de respuesta individual. Los alumnos estaban acostumbrados a realizar Kahoots (es una herramienta que es utilizada en casi todas las clases, aunque en su modalidad competitiva de quizz).

Seguidamente los alumnos se dividieron en 8 grupos de trabajo (los grupos fueron creados por ellos mismos al comienzo de curso para la realización de diversas actividades programadas durante el cuatrimestre), para el análisis de 2 microcasos de teoría de juegos. Se elige un/a secretario/a por grupo, que toma nota de lo decidido en cada microcaso. El primero de ellos coincidía con uno de los presentados en la encuesta de Kahoot (pregunta 6), el otro no. En concreto se trataba de la subasta (a una solo puja) del billete de $1 \$$ en donde ganaba el mejor postor, pero también tenía que pagar la segunda mejor puja sin llevarse nada. Es decir, se trata de un claro juego no equitativo, en el que la mejor jugada de cualquier jugador es NO APOSTAR. Es reseñable cómo la dinámica de resolución del microcaso en grupo supuso que desaparecieran todas las respuestas correctas a este problema. En los 8 grupos se impusieron los razonamientos no correctos, a pesar de que en encuesta inicial con Kahoot hubo un 34\% de respuestas correctas. De esta sesión se analizan los datos de las encuestas y de la dinámica del grupo para extraer conclusiones.

Sesión 2a y 3o (120 minutos + 120 minutos)

En estas 2 sesiones se realizaron un total de 8 microcasos de juegos. La dinámica aplicada fue la siguiente: los alumnos se dividieron en sus 8 grupos de trabajo en clase. Se les planteaba el microcaso y se les dejaba un periodo de 10-15 minutos (según complejidad) para el análisis, debate y resolución. A continuación iniciábamos un debate general, en el que el profesor actuaba de moderador, las conclusiones sobre el microcaso se apuntaban en la pizarra. Seguidamente, el profesor presentaba el contenido 
teórico con transparencias PowerPoint, se resolvía el microcaso de forma correcta y finalmente se abría un debate sobre el microcaso y los errores detectados. Resultó especialmente interesante el hecho de que los propios alumnos descubrian en el debate final cuales habían sido sus esquemas erróneos de pensamiento en la respuesta consensuada en la dinámica de grupo y por qué.

Sesión 4ạ (120 minutos)

En la última sesión, comenzamos haciendo de nuevo la Encuesta Individual de 9 preguntas de la Sesión 1a. Se utilizó también la herramienta Kahoot, en su modalidad de encuesta (survey) y en el modo de respuesta individual. Una vez concluida la encuesta, se procedió a ir proyectando uno a uno los 4 microcasos, iniciándose un debate abierto entre toda la clase sobre las posibles soluciones. El profesor actuó como moderador, apuntando inicialmente en la pizarra las respuestas de los alumnos y abriendo el debate con preguntas incisivas sobre los aspectos claves a considerar y a valorar para lograr una respuesta correcta.

\section{Evaluación del aprendizaje}

\section{a) Preguntas intrigantes:}

\section{a.1.a) Pregunta 6.}

Las Figura 3 y 4 presentan los resultados obtenidos (en número absoluto y \% relativo) de la Pregunta 6 (Subasta de un billete de $1 \$$, a una sola puja. El mejor postor se lleva el billete. El segundo mejor postor también paga su oferta. ¿Cuánto apuestas?: 0,5\$/1\$/0,25\$/Nada), del cuestionario Inicial (38 alumnos) y Final (36 alumnos).

Respuesta correcta: NADA. Respuestas correctas Cuestionario Inicial 34 \%. Respuestas correctas Cuestionario Final $89 \%$ 
Subasta billete 1 \$: Mejor postor se lleva el billete. El segundo mejor postor también paga su oferta¿Cuanto apuestas?

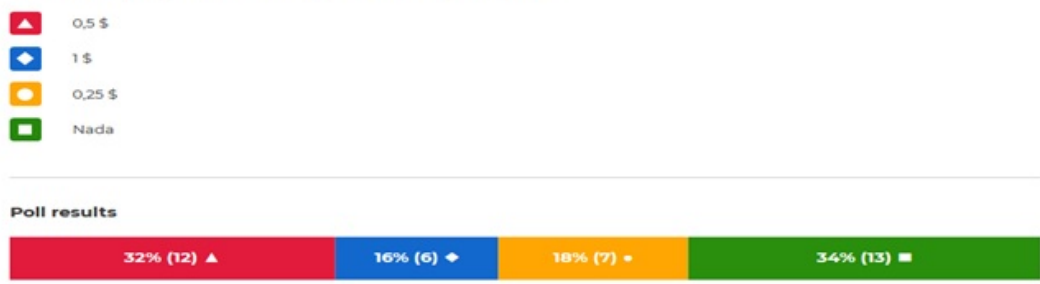

Figura 3. Pregunta 6. Cuestionario Inicial con respuestas obtenidas

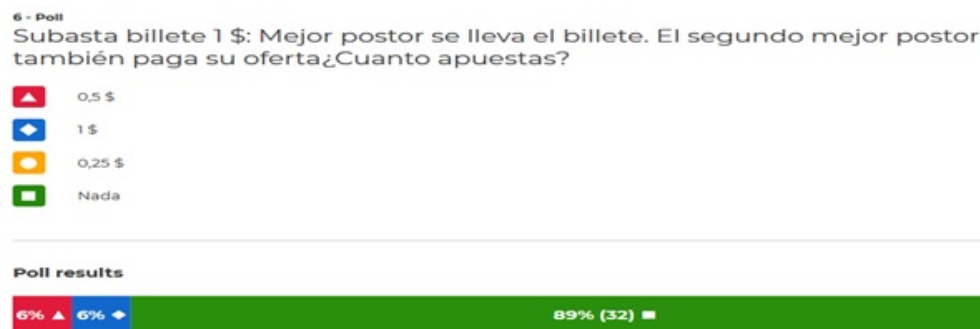

Figura 4. Pregunta 6. Cuestionario Final con respuestas obtenidas a.1.b) Pregunta 6. Valoración del Aprendizaje Alcanzado

Este primer reto intrigante, trataba de enseñar a los alumnos la necesidad de analizar la equidad de un juego antes de iniciarlo. Comparando las escaleras inicial y final (Figura 5), observamos que el objetivo muestra una evolución positiva de 55\% (de 13 a 32 alumnos).

\begin{tabular}{|c|c|c|c|}
\hline Escalera Inicial & $\begin{array}{l}\text { Nivel 1: } \\
\text { Rechazar } \\
\text { cualquier } \\
\text { apuesta (34\%) }\end{array}$ & Escalera Final & $\begin{array}{l}\text { Nivel 1: } \\
\text { Rechazar } \\
\text { cualquier } \\
\text { apuesta }(89 \%)\end{array}$ \\
\hline $\begin{array}{l}\text { Nivel 0: } \\
\text { Aceptar } \\
\text { cualquier } \\
\text { apuesta }(66 \%)\end{array}$ & $\begin{array}{l}\text { No analiza la } \\
\text { Equidad del } \\
\text { Juego }\end{array}$ & $\begin{array}{l}\text { Nivel 0: } \\
\text { Aceptar } \\
\text { cualquier } \\
\text { apuesta (11\%) }\end{array}$ & $\begin{array}{l}\text { No analiza la } \\
\text { Equidad del } \\
\text { Juego }\end{array}$ \\
\hline
\end{tabular}

Figura 5. Escaleras Inicial y Final pregunta 6

\section{b.1.a) Pregunta 7.}

Las Figura 6 y 7 presentan los resultados obtenidos (en número absoluto y \% relativo) de la Pregunta 7 (Se plantea una matriz de beneficios para 2 empresas A y B que presentan 2 estrategias (hacer publicidad, no hacer 
publicidad): 10,5/ 15,0/ 6,8/ 20,2), del cuestionario Inicial (38 alumnos) y Final (36 alumnos).

Respuesta correcta: A y B HACEN PUBLICIDAD. Respuestas correctas Cuestionario Inicial $37 \%$. Respuestas correctas Cuestionario Final 53\%

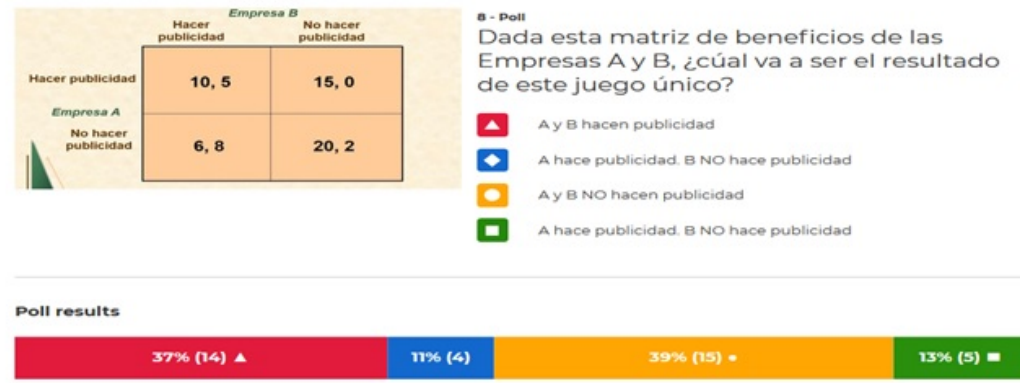

Figura 6. Pregunta 7. Cuestionario Inicial con respuestas obtenidas

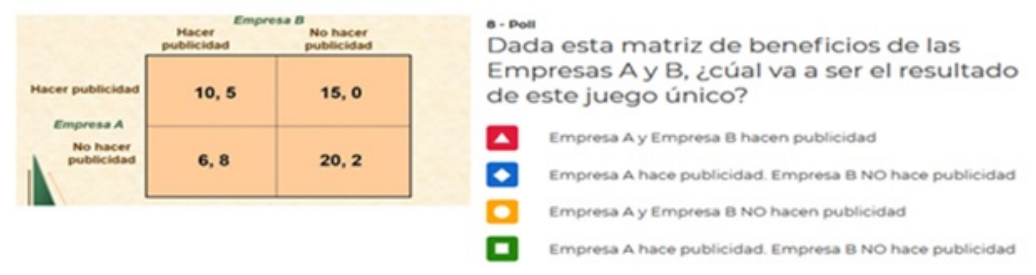

Poll results

Figura 7. Pregunta 7. Cuestionario Final con respuestas obtenidas

\section{b.2.b) Pregunta 7.Valoración del Aprendizaje Alcanzado}

En este problema intrigante nos encontramos en un Equilibrio de Nash en el que la Empresa A depende de lo que haga la B. Dado que la Empresa B tiene una estrategia dominante de hacer publicidad, el resultado final es que ambas, A y B, hacen publicidad. En la Figura 8 podemos apreciar que la evolución en el aprendizaje de los alumnos es muy positiva, los alumnos que analizan las consecuencias del juego pasan de 9 a $11(+7 \%)$ y los que tienen en consideración las acciones conjuntas de las dos empresas pasan de 14 a $19(+16 \%)$. 


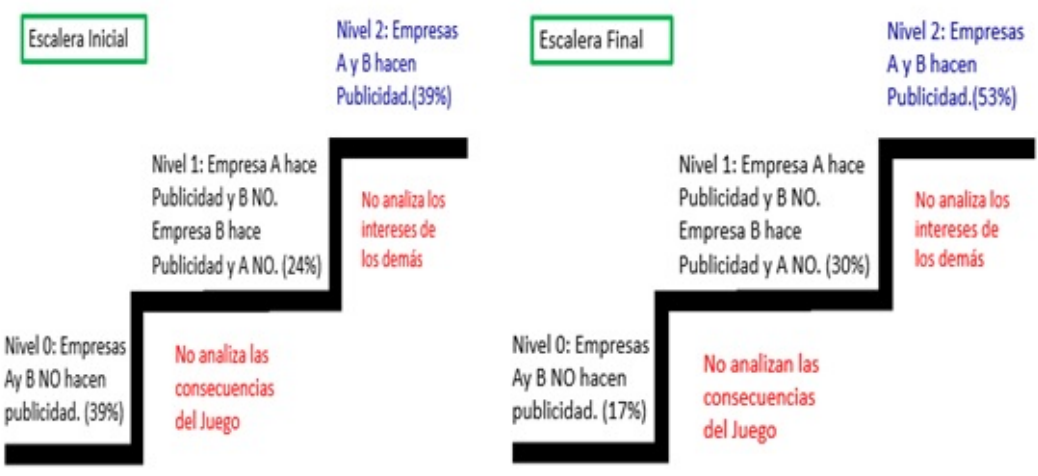

Figura 8. Escaleras Inicial y Final pregunta 7

\section{c.3.a) Pregunta 8.}

Las Figura 9 y 10 presentan los resultados obtenidos (en número absoluto y \% relativo) de la Pregunta 8 (En esta plaza rectangular se van a emplazar el Bar Pepe y el Bar Paco, ¿dónde se situarán para maximizar beneficios?: uno frente al otro/ los dos pegados/ uno en medio y el otro en la esquina/ Ninguno es correcto), del cuestionario Inicial (38 alumnos) y Final (36 alumnos).

Respuesta correcta: En 3 (uno frente al otro). Respuestas correctas Cuestionario Inicial 29 \%. Respuestas correctas Cuestionario Final 33\%

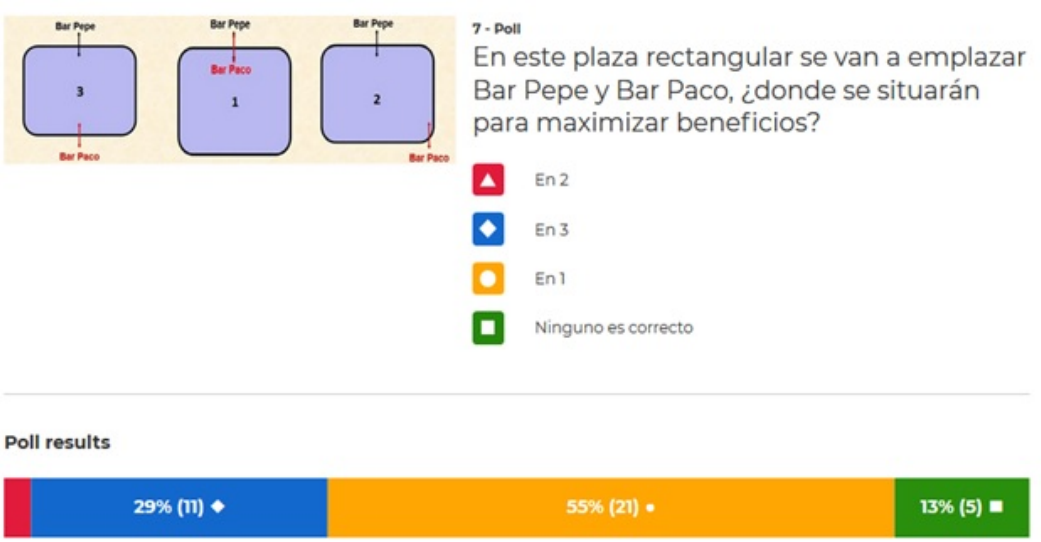

Figura 9. Pregunta 8. Cuestionario Inicial con respuestas obtenidas

Jornadas de Formación e Innovación Docente del Profesorado | № 2 (2019) Esta obra se distribuye con la licencia Creative Commons Reconocimiento-NoComercial-SinObraDerivada 4.0 Internacional (CC BY-NC-ND 4.0.) 
a ser resultado de este juego repetido?: 10,10/ 100, -50/ $-50,100 / 50,50)$, del cuestionario Inicial (38 alumnos) y Final (36 alumnos).

Respuesta correcta A y B Precio Alto. Respuestas correctas Cuestionario Inicial 53\%. Respuestas correctas Cuestionario Final 53\%

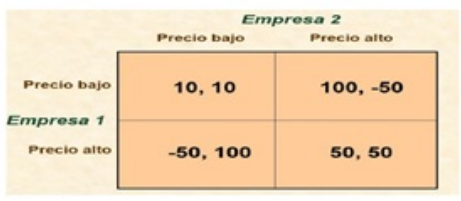

Dada esta matriz de beneficios de las

Empresas Ay B. ¿cúal va a ser el resultado

de este juego REPETIDO?

4 Aув Precio bajo

- A Precio alto B Precio bajo

-1. Ay B Precio alto

- A Precio bajo. B Precio alto

Poll results

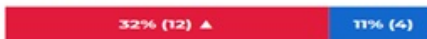

Figura 12. Pregunta 9. Cuestionario Inicial con respuestas obtenidas

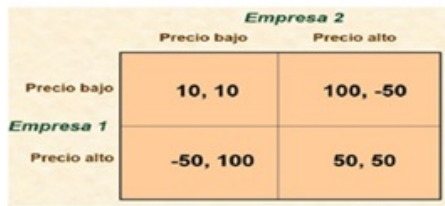

Dada esta matriz de beneficios de las

Empresas A y B, ¿cúal va a ser el resultado de este juego REPETIDO?

mpresa 1 y Empresa 2 Precio bajo

당

Empress 1 Precio alto Empress 2 Precio bajo

임

Empresa 1 Y Empresa 2 Precio ato

- Empresa 1 Precio bajo Empresa 2 Precio alto

Poll results

$19 \times(7) \wedge$

Figura 13. Pregunta 9. Cuestionario Final con respuestas obtenidas

c.4.b) Pregunta 9. Valoración del Aprendizaje Alcanzado

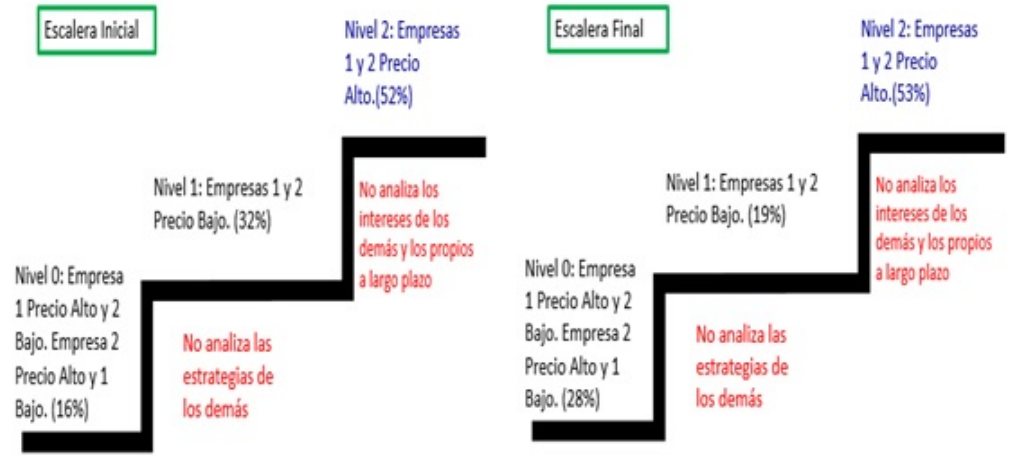

Figura 14. Escaleras Inicial y Final pregunta 9

Jornadas de Formación e Innovación Docente del Profesorado | № 2 (2019) Esta obra se distribuye con la licencia Creative Commons Reconocimiento-NoComercial-SinObraDerivada 
En el último problema intrigante se cuestionó sobre el resultado en un juego repetido. Es decir, había que proyectar la estrategia de comportamiento de los jugadores cuando el juego se realiza infinitas veces. En esta ocasión los resultados son levemente negativos (Figura 14), dado que se pasa de 20 a 19 los alumnos que dan la respuesta correcta, aunque el porcentaje relativo es igual, dado que la encuesta final la realizaron 2 alumnos menos que la inicial.

En conclusión, el análisis y comparativa de los resultados de las Encuestas Inicial y Final de los alumnos muestra un avance en el conocimiento en tres de los cuatro retos planteados. El nivel del avance en el conocimiento está en conexión directa con el nivel de dificultad del concepto a asimilar, es decir, ha sido menor en las preguntas intrigantes más complicadas.

Resulta especialmente interesante la cognición que se logra sobre los aspectos que resultan dificiles de asimilar a los alumnos. La realización de la encuesta inicial ha permitido incluso graduar y cuantificar este grado de dificultad.

\section{Evaluación del Ciclo de Mejora. Conclusiones y discusión}

La aplicación del Ciclo de Mejora me ha permitido como docente comprender mejor el modelo de pensamiento de los alumnos respecto al contenido motivo de aprendizaje. La Encuesta Inicial y las actas de los grupos de la primera sesión, permitieron definir los puntos críticos de dificultad que encontraban los alumnos durante el proceso de aprendizaje. Es reseñable cómo la dinámica de grupo se vuelve un instrumento de imposición de la opinión de la mayoría, lo cual puede ocultar opiniones disconformes más cercanas a la solución correcta. 
La creación del ambiente adecuado en las sesiones 2a y 3a para un aprendizaje crítico natural a través de la resolución de microcasos reales en equipos de trabajo, ha sido una experiencia especialmente gratificante. La posterior explicación del contenido teórico y, particularmente, el análisis en dinámica de debate, de las conclusiones erróneas inicialmente alcanzadas en los equipos de trabajo, han permitido una profundización en la materia y en la manera de asimilarla.

La metodología docente cognitivista y constructivista, aplicando Aprendizaje Basado en Problemas (ABP) a través de un taller conceptual materializado en microcasos parece presentar serias ventajas. De una parte, la aceptación y participación activa del alumnado. Por otro lado los resultados de la valoración final de los aprendizajes alcanzados son positivos y esperanzadores, por lo que animan a continuar aplicando sucesivamente esta metodología en los demás contenidos teóricos de la asignatura.

Jornadas de Formación e Innovación Docente del Profesorado I № 2 (2019) Esta obra se distribuye con la licencia Creative Commons 


\section{Referencias}

Finkel, D. (2008). Dar clase con la boca cerrada. Valencia: Públicaciones Universidad de Valencia.

Bain, K. (2005). Lo que hacen los mejores profesores universitarios. Valencia: Universitat de Valencia.

García, J. E. (1998). Hacia una teoría alternativa sobre los contenidos escolares. Sevilla: Diada.

Nash, John F. (1950). Non-cooperative games. Tesis de doctorado. Departamento de Matematicas, Princeton University.

Porlán, R. (2017). Enseñanza universitaria. Cómo mejorarla. Madrid: Morata.

Von Neumann, J., Morgenstern, O., \& Kuhn, H. W. (2007). Theory of games and economic behavior (commemorative edition). Princeton university press.

Jornadas de Formación e Innovación Docente del Profesorado | № 2 (2019) Esta obra se distribuye con la licencia Creative Commons 